Sir,

\section{Ciliary effusion complicates blunt ocular trauma} Eye (2003) 17, 835-836. doi:10.1038/sj.eye.6700473

Common complications of blunt ocular injury in the anterior segment of the eye include traumatic hyphema, iritis, and mydriasis. Previously, a small number of cases of blunt ocular trauma complicated by ciliary effusion have been described. In 1982, Dotan and Oliver ${ }^{1}$ reported three cases with shallow anterior chamber and uveal effusion after ocular blunt injuries, diagnosed by indirect ophthalmoscope. More recently, two cases of ciliary effusion with traumatic myopia were presented by a Japanese group. ${ }^{2}$ Here, we describe a case of ciliary effusion with hypotony and transient myopia induced by blunt ocular injury.

\section{Case report}

We treated a 37-year-old man who complained of blurred vision in his right eye, which had been hit by a small piece of plastic material several hours prior. Visual acuity without correction in the affected eye was 20/400. A mildly injected conjunctiva was noted. Slit-lamp examination revealed a deep anterior chamber with $3+$ cells. The intraocular pressure (IOP) was $9 \mathrm{~mm} \mathrm{Hg}$. Indirect ophthalmoscopy showed commotio retinae at the temporal periphery. Visual acuity without correction in the left eye was 20/30. Slit-lamp and ophthalmoscopic findings of the left eye were normal, with an IOP of $10 \mathrm{~mm} \mathrm{Hg}$.

By the next day, when the patient returned to the outpatient department for follow-up, slit-lamp examination of the right eye revealed a very shallow anterior chamber with anterior shift of the lens. There were $2+$ cells in the anterior chamber. The IOP had fallen to $5 \mathrm{~mm} \mathrm{Hg}$ in the right eye, but was $12 \mathrm{~mm} \mathrm{Hg}$ in the left eye. Visual acuity without correction was 20/100 and the best corrected visual acuity was 20/20 with sph -2.0 D. The patient denied having previously experienced refractive error Ultrasound biomicroscopy (UBM) revealed ciliary effusion in all four quadrants. In addition, anterior rotation, thickening of the ciliary processes, and iris bombé were observed (Figure 1a, b). An ultrasound B-scan disclosed no abnormal findings in the posterior part of the eye. The patient was treated with prednisolone $5 \mathrm{mg}$ orally four times a day as well as $1 \%$ prednisolone acetate and $2 \%$ cyclopentolate instilled four times a day.

After 7 days, the IOP in the right eye had returned to $10 \mathrm{mmHg}$. UBM revealed a deeper anterior chamber, and residual ciliary effusion was found only over the superior and temporal parts (Figure 1c, d). Another 3 days later, visual acuity without correction had returned to 20/30, and no further ciliary effusion was found by UBM. Oral prednisolone was then discontinued.

\section{Comments}

Ocular disorders such as tumours, nanophthalmos, arteriovenous fistula, and ocular inflammation following panretinal photocoagulation or intraocular surgery are associated with ciliochoroidal effusion. Ciliary effusion without obvious choroidal effusion cannot be detected by slit-lamp, gonioscopy, or fundus examination. UBM provides information about the anterior segment, such as the presence of foreign bodies, lens dislocation, angle recession, iridodialysis, cyclodialysis, or ciliary effusion, and the procedure is relatively noninvasive for trauma patients.

The relation between ciliochoroidal detachment and hypotony is not clearly understood. Based on previous studies, ${ }^{3,4}$ it was suggested that hypotony perpetuates detachment, which in turn exacerbates hypotony. During hypotony, the pressure drop from the suprachoroidal space to the outer scleral surface is lower, favouring fluid accumulation in the suprachoroidal space. On the other hand, ciliochoroidal effusion causes hyposecretion of the ciliary body. It has also been concluded that ciliochoroidal effusion enhances uveoscleral outflow.

In our case, traumatic iridocyclitis appeared to be responsible for both ciliary effusion and hypotony. Iridocyclitis had the dual effect of decreasing aqueous humour formation, leading to hypotony, and of increasing colloidal leakage into the subciliary space and causing ciliary effusion. Ciliary effusion also increased excretion of aqueous humour through the uveoscleral route. Once inflammation had been diminished by steroid treatment, the cycle of ciliary effusion and hypotony was broken.

A number of potential causes of transient traumatic myopia have been proposed, such as: (1) ciliary spasm, ${ }^{5}$ (2) anterior displacement of the lens-iris diaphragm induced by ciliary effusion, ${ }^{2,6}$ and (3) increased thickness of the crystalline lens. ${ }^{7}$ We believe that the cause of myopic shift in this patient was an anterior shift of the lens induced by ciliary effusion. The displaced anterior lens has greater dioptric power. In this patient, a deeper anterior chamber correlated with better uncorrected visual acuity. Also, cycloplegics were prescribed on the first day although myopic shift and ciliary effusion were still occurring. Previous reports describing a hyperopic shift after cycloplexy for cyclodialysis patients also support this speculation. ${ }^{8}$

In conclusion, we reported a rare case of traumatic ciliary effusion with a shallow anterior chamber, hypotony, and myopic shifting. With a correct diagnosis 

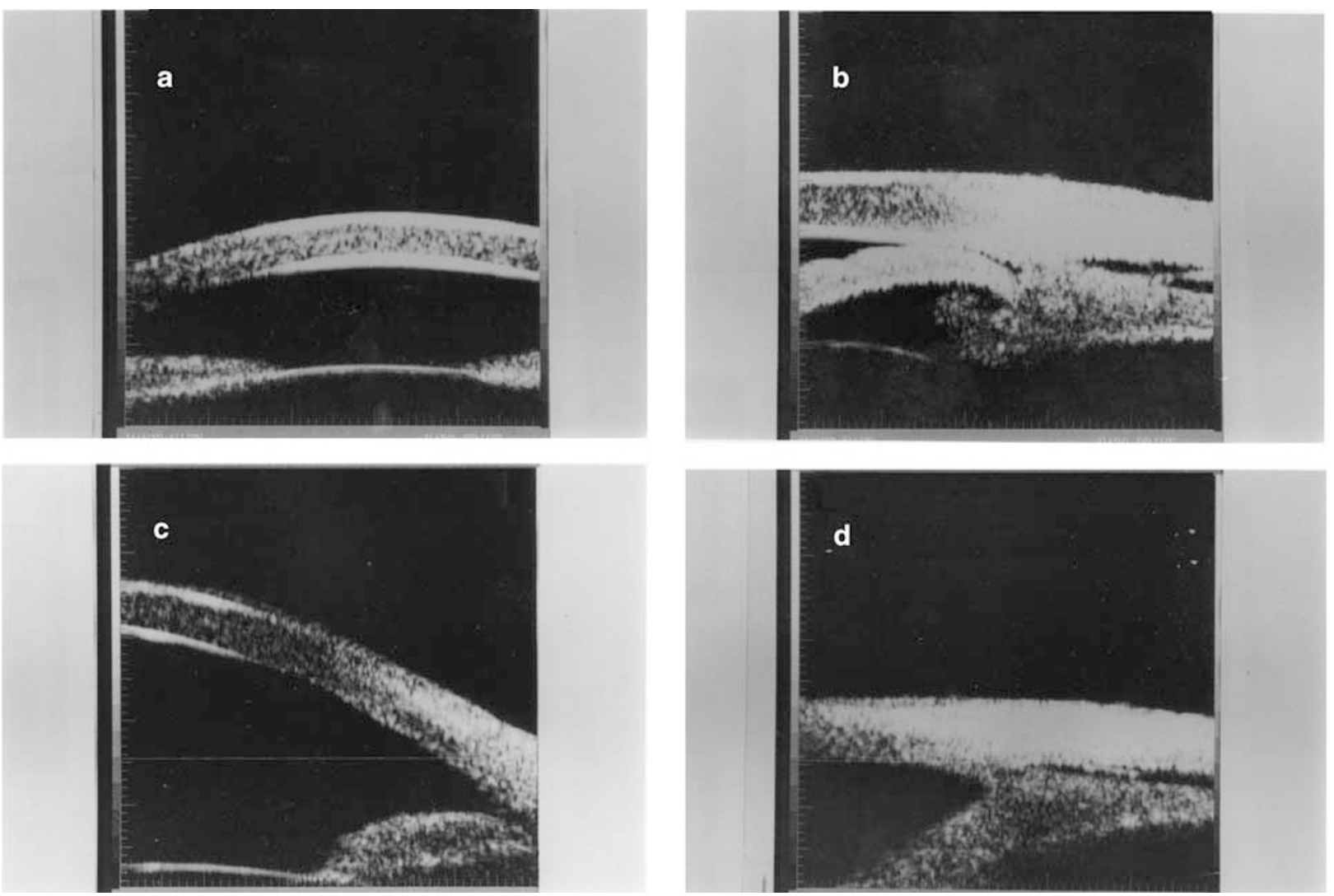

Figure 1 UBM of the affected eye. (a) The anterior chamber was shallow, with a central depth of $1.3 \mathrm{~mm} 1$ day after blunt ocular injury. (b) Ciliary effusion with anterior rotation, thickening of the ciliary processes, and iris bombé were noted. After 7 days, (c) a deeper anterior chamber and (d) partial resolution of the subciliary fluid were noted.

through UBM and through treatment with steroids, the cycle of hypotony and ciliary effusion was broken and the patient recovered.

\section{References}

1 Dotan S, Oliver M. Shallow anterior chamber and uveal effusion after nonperforation trauma to the eye. Am J Ophthalmol 1982; 94: 782-784.

2 Ikeda N, Ikeda T, Nagata M, Mimura O. Pathogenesis of transient high myopia after blunt eye trauma. Ophthalmology 2002; 109 (3): 501-507.

3 Richard FB, Jonathan EP. Ciliochoroidal detachment. Surv Ophthalmol 1983; 27: 281-289.

4 Salvatore D, Chharles LS. Can chronic bulbar hypotony be responsible for uveal effusion? Report of two cases. Ophthalmic Surg 1989; 20: 872-875

5 Duke-Elder S. System of Ophthalmology, Vol 5 Kimpton: London, 1970, pp 354-355.

6 Kraus E, Meyer E, Zonis S. Traumatic myopia due to ciliary body detachment. Harefuah 1989; 117 (7-8): 191-192.

7 Steele CA, Tullo AB, Marsh IB, Storey JK. Traumatic myopia; an ultrasonographic and clinical study. Br J Ophthalmol 1987; 71: $301-303$
8 Kuchle M, Nammann GO. Direct cycloplexy for traumatic cyclodialysis with persisting hypotony. Ophthalmology 1995; 102: 322-333.

\section{H-C Chen, SH-L Chang, S-N Chen and J-D Ho}

\section{Department of Ophthalmology}

Chang-Gung Memorial Hospital

2F 23-9 Ching Chang Street

Taipei, Taiwan

Correspondence: J-D Ho

Tel: $+88633281572 ;+88633281200$ ext. 8666

Fax: +88633281572

E-mail: jdho@seed.net.tw

The authors have no propriety interest in any of the material used in this study. 